\title{
Letter
}

\section{Fractional Dynamics of Cancer Cells and the Future of Research in Biomedicine}

\author{
Hosein Nasrolahpour \\ Department of Physics, School of Sciences, Tarbiat Modares University, Tehran, Iran
}

Email address:

hnasrolahpour@gmail.com

\section{To cite this article:}

Hosein Nasrolahpour. Fractional Dynamics of Cancer Cells and the Future of Research in Biomedicine. Cancer Research Journal. Vol. 6, No. 1, 2018, pp. 16-19. doi: 10.11648/j.crj.20180601.13

Received: December 5, 2017; Accepted: December 13, 2017; Published: January 12, 2018

\begin{abstract}
Following our previous works on fractional biophysical issues such as fractional dynamics of protein folding process and fractional dynamics of cancer cells and their branching processes, in this work we further develop these issues and propose a new fractional biomechanics of cancer cells. In this short note we present some promising models for future studies in biomedicine, including constant and variable order fractional Maxwell and Kelvin-Voigt models to study the mechanics of cancer cells. We also emphasize that fractional calculus will play a vital and central role in the understanding of the complexities that occur when we deal with the phenomena and processes in the realm of bioscience and biomedicine and particularly in physics of cancer.
\end{abstract}

Keywords: Fractional Dynamics, Complex Systems, Fractional Biomechanics of Cancer Cells

\section{Introduction}

The study of complex systems and the investigation of their structural and dynamical properties have attracted considerable interest among scientists in general and physicists and biologists in particular. Complex systems can be found almost everywhere, including: financial markets, highway transportation networks, telecommunication networks, social networks, computational systems however the highest level of complexities are related to living and biological organisms and systems. Complex systems are often composed of large numbers of interconnected and interacting entities whose interactions lead to emergent collective behaviors and in particular exhibit the emergence of self-organization.

As a physicist we always are able to model natural phenomena using systems of differential equations and nowadays it is well know that the advantage of fractional-order differential equation systems over ordinary differential equation systems is that they are more comprehensive and also incorporate memory effect in the model [1-28] and due to this fact they have found many applications in the realm of bioscience and biomedicine to understand the emergence of complexities in bio-structures and living systems. In the field of fractional calculus we use new concepts of fractional integral and fractional derivative. The kernel function of fractional derivative is called memory function [29] that is very useful to describe the complex dynamics of complex systems. Recently it is showed that the fractional model perfectly fits the test data of memory phenomena in different disciplines [30] they have found that a possible physical meaning of the fractional order is an index of memory. From this viewpoint fractional calculus has found many applications in new research on physics of biological structures and living organisms, from DNA dynamics [31-33] to protein folding [34] (in this work we have presented a coupled system of fractional differential equations for the folding process of protein as follows:

$$
\begin{aligned}
\eta(\mathrm{t})^{2(\alpha-1)} \partial_{-} \partial_{t}^{\alpha}\left({ }_{+}^{C} \partial_{t}^{\alpha} u\right) & =\eta(x)^{2(\alpha-1)}{ }_{-} \partial_{x}^{\alpha}\left({ }_{+}^{C} \partial_{x}^{\alpha} u\right) \\
& -m_{u}^{3 \alpha} / \sqrt{\lambda_{u}} \sin \left(\sqrt{\lambda_{u}} u / m_{u}\right) \\
& +2 \Lambda_{\alpha} u v^{2}
\end{aligned}
$$




$$
\begin{aligned}
\eta(\mathrm{t})^{2(\alpha-1)}{ }_{-} \partial_{t}^{\alpha}\left({ }_{+}^{C} \partial_{t}^{\alpha} v\right) & =\eta(x)^{2(\alpha-1)}{ }_{-} \partial_{x}^{\alpha}\left({ }_{+}^{C} \partial_{x}^{\alpha} v\right) \\
& -\left(\lambda_{v}^{\alpha} / 6\right) v^{3}+2 \Lambda_{\alpha} v u^{2}
\end{aligned}
$$

(for details refer to the mentioned paper) as we have mentioned in this work, this model is the most comprehensive model such that all previous models can be derived from it), cancer cells [35], tumor-immune system [36], modeling of some human autoimmune diseases such as psoriasis [37], bioimpedance [38], spiking neurons [39], and also the transport of drugs across biological materials and human skin [40] and electrical impedance applied to human skin [41] and even modeling of HIV dynamics [42].

In our previous work we have purposed new approach of fractional calculus to understand the physics behind the cancer cells dynamics [43]. In this work we have investigated cancer growth process in the framework of fractional dynamics and we have obtained new results. In the mentioned article we have proposed new model based and fractional calculus for branching processes which are a class of simple models that have been used extensively to model growth dynamics of stem cells and cancer cells. We have also presented a new model for the average colony size $\langle s\rangle$ as follows:

$$
\begin{aligned}
\eta^{(\alpha-1)} \frac{d^{\alpha}\left\langle s_{\alpha}(t)\right\rangle}{d t^{\alpha}} & =\eta^{(\alpha-1)}{ }_{0}^{c} D_{t}^{\alpha}\left\langle s_{\alpha}\right\rangle \\
& =(\varphi-\beta)\left\langle s_{\alpha}\right\rangle
\end{aligned}
$$

with general solution in terms of the well-known one-parameter Mittag-Leffler function in the form of:

$$
\left\langle s_{\alpha}(t)\right\rangle=\langle s\rangle_{0} E_{\alpha}\left(-\eta^{1-\alpha} t^{\alpha}((\beta-\varphi))\right)
$$

(for details refer to the mentioned paper). In the present work we want to propose a new fractional biomechanics of cancer and further develop the new field of fractional dynamics of cancer cells.

For these purpose in the following: concepts of fractional dynamics are briefly reviewed in Sec. 2. Then in Sec. 3 we introduce fractional viscoelastic model. Based on the fractional viscoelastic model, fractional biomechanics of cancer cells is presented in this section. At last, in Sec. 4, we will present some conclusions.

\section{Fractional Dynamics}

Fractional dynamics is a field in theoretical and mathematical physics, studying the behavior of objects and systems that are described by using integrations and differentiation of fractional orders, i.e., by methods of fractional calculus. Derivatives and integrals of non-integer orders are used to describe objects that can be characterized by: (1) a power-law non-locality (2) a power-law long-term memory (3) a fractal-type property [44]. As an example in the realm of classical physics we can consider the well-known diffusion phenomena. The most known diffusion processes is the normal diffusion. This process is characterized by a linear increase of the mean squared distance:

$$
\left\langle r^{2}(t)\right\rangle \propto t
$$

where $r$ is the distance a particle has traveled in time $t$ from its starting point. However there are many examples of phenomena in the natural sciences that violate this kind of behavior i.e. they are slower or faster than normal diffusion. In these cases (anomalous diffusions) the mean squared displacement is no longer linear in time:

$$
\left\langle r^{2}(t)\right\rangle \propto t^{\alpha}, \quad 0<\alpha<2
$$

In recent years it is well known that generalization of the well-known diffusion equation and wave equation such that it includes derivatives of non-integer order with respect to time can describes phenomena that satisfy such a power law mean squared displacement. The fractional diffusion-wave equation [44] is the linear fractional differential equation obtained from the classical diffusion or wave equations by replacing the firstor second-order time derivatives by a fractional derivative (in the Caputo sense) [45-48] of order $\alpha$ with $0<\alpha<2$,

$$
\nabla^{2} u(t, x)=C_{\alpha}{ }_{0}^{C} D_{t}^{\alpha} u(t, x)
$$

This equation describes diffusion-wave phenomena $[49,50]$ which is also called the anomalous diffusion such that we have the super-diffusion for $1<\alpha<2$, and sub-diffusion for $0<\alpha<1$. In above equations the fractional derivative of order $\alpha, n-1<\alpha<n, n \in N$ is defined in the Caputo sense:

$$
\begin{aligned}
{ }_{0}^{c} D_{t}^{\alpha} f(t) & =\frac{\partial^{\alpha} f(t)}{\partial t^{\alpha}} \\
& =\frac{1}{\Gamma(n-\alpha)} \int_{0}^{t}(t-\tau)^{n-\alpha-1} \frac{\partial^{n} f(\tau)}{\partial \tau^{n}} d \tau
\end{aligned}
$$

Where $\Gamma$ denotes the Gamma function. For $\alpha=n, n \in N$ the Caputo fractional derivative is defined as the standard derivative of order $n$. It is also worth noting that we mention the order of fraction derivative can be constant or variable. To derive a solution for a process described by an equation containing Caputo fractional derivatives, we need the initial conditions that can be written as:

$$
f^{(k)}(0)=c_{k} \quad, k=0,1, \ldots,(n-1)
$$

and because of this point that we are seeking the causal solution for natural phenomena we require that $f(t)=0$ for $t<0$.

\section{Fractional Biomechanics of Cancer}

Fractional calculus has been considered as a powerful tool to model physical responses and is particularly suitable for building the time-dependent constitutive model. The use of the fractional calculus is motivated in large part by the fact that fewer parameters are required to achieve accurate approximation of experimental data [51]. It is well known that the ideal solid obeys the Hooke's law, $\sigma(t) \propto \varepsilon(t)$, and a Newtonian fluid satisfies the Newton's law of viscosity, 
$\sigma(t) \propto d \varepsilon(t) / \mathrm{dt}$, where $\sigma$ and $\varepsilon$ are the stress and strain. So it is not difficult to imagine that the intermediate material, which is intermediate between ideal solid and Newtonian fluid should follow [51]:

$$
\sigma(t)=E \eta^{(\alpha-1)} \frac{d^{\alpha} \varepsilon(t)}{d t^{\alpha}}
$$

where $E$ is Young modulus and $\eta$ is an arbitrary quantity with dimension of [second] to ensure that all quantities have correct dimensions and also $0 \leq \alpha \leq 1$, where we have the pure elasticity for $\alpha=0$, and the pure viscosity for $\alpha=1$ [see the Ref. 51 and references therein]. Fractional viscoelastic models have found many application in bioscience and biomedicine during the last decade [52-55]. In the next section we will present fractional approach to study the biomechanics of cancer cells.

Like any other material, cells respond to mechanical perturbations by deforming, but unlike passive elastic objects, however, they can apply active forces to the environment. Active matter is attracting a large amount of attention in the field of soft matter, with many results having direct relevance to cell mechanics [56].

Cells are extremely soft materials, with a Young modulus which can be measured by different means such as, for example, atomic force microscopy. Cells, however, are not simple Hookean solids since their mechanical response is time-dependent and include a viscous component typical of fluids [56]. The simplest description of viscoelasticity is in terms of springs and dashpots that can be combined in series, leading to the Maxwell model, which in its scalar version can be written as:

$$
\frac{d \varepsilon}{d t}=\frac{\sigma}{\tilde{\eta}}+\frac{1}{E} \frac{d \sigma}{d t}
$$

where $\tilde{\eta}$ is the viscosity, or in parallel, leading to the KelvinVoigt model:

$$
\sigma=E \varepsilon+\tilde{\eta} \frac{d \varepsilon}{d t}
$$

The Maxwell and Kelvin-Voigt models predict exponential relaxations of strain and stress, respectively, with a characteristic time $\tau=E / \tilde{\eta}$. Relaxation in cells is not exponential, as predicted by these models, but decays as a power law. In particular, the time-dependent response to a constant applied stress $\sigma$, or creep, follows [56]:

$$
\varepsilon(t)=\sigma\left(t / t_{0}\right)^{\beta}
$$

Similarly, the stress relaxation in response to a fixed deformation decays as a power law at long timescales:

$$
\sigma(t) \approx t^{-\alpha}
$$

while at short timescales one observes deviations attributed to the poroelastic behavior of the cell. Viscoelastic behavior is common to many polymeric materials, but cells are different because their response to stress is not only passive but contains an active component due to acto-myosin-driven contraction of the cytoskeleton [56]. This behavior can be described in the best way using fractional versions of Maxwell and Kelvin-Voigt models. Here we propose two versions of these models:

I- Fractional Maxwell and Kelvin-Voigt models in term of constant order fractional derivative:

$$
\begin{gathered}
\eta^{(\alpha-1)} \frac{d^{\alpha} \varepsilon}{d t^{\alpha}}=\frac{\sigma}{\tilde{\eta}}+\frac{\eta^{(\beta-1)}}{E} \frac{d^{\beta} \sigma}{d t^{\beta}} \quad, 0 \leq \alpha, \beta \leq 1 \\
\sigma=E \varepsilon+\tilde{\eta} \eta^{(\alpha-1)} \frac{d^{\alpha} \varepsilon}{d t^{\alpha}}, 0 \leq \alpha \leq 1
\end{gathered}
$$

II- Fractional Maxwell and Kelvin-Voigt models in term of variable order fractional derivative:

However it has been found that some mechanical behaviors of cells still cannot be fully understand by above equations. The possible reason can be that the constant fractional order in Eqs. $(15,16)$ implicates the invariability of mechanical property while in the real living organisms and systems it is changing during the mechanical process. A further generalization of the concept of fractional order calculus that is applicable to more complex mechanical property of material is that of a calculus of varying order [51].

$$
\begin{gathered}
\eta^{(\alpha(t)-1)} \frac{d^{\alpha(t)} \varepsilon}{d t^{\alpha(t)}}=\frac{\sigma}{\tilde{\eta}}+\frac{\eta^{(\beta(t)-1)}}{E} \frac{d^{\beta(t)} \sigma}{d t^{\beta(t)}}, 0 \leq \alpha(t), \beta(t) \leq 1 \\
\sigma=E \varepsilon+\tilde{\eta} \eta^{(\alpha(t)-1)} \frac{d^{\alpha(t)} \varepsilon}{d t^{\alpha(t)}} \quad, 0 \leq \alpha(t) \leq 1
\end{gathered}
$$

\section{Conclusion}

Living organisms and systems exhibits the highest level of complexities. The aim of this work was only presentation of some promising models for the future studies in biomedicine based on the power tool of fractional calculus. Nowadays it is understood that the best tool for investigation of physics of such systems is fractional calculus. We believe that we will have exact information about biological phenomena and also more predictable behavior of biological structures using fractional calculus in future. Based on this motivation in this work we have proposed new versions of Maxwell and Kelvin-Voigt models that is constant and variable order fractional Maxwell and Kelvin-Voigt models, to study the complex behavior and mechanics of cancer cells. Finally we emphasize that fractional calculus will play a vital and central role in the understanding of the complexities that occur when we deal with the phenomena and processes in the realm of bioscience and biomedicine and particularly in physics of cancer and the models presented in this work will have a promising future. 


\section{References}

[1] R. Gorenflo and F. Mainardi, Fractional Calculus (Springer, 1997).

[2] H. Nasrolahpour, Prespacetime J. 2 (8) (2011) 1264-1269.

[3] H. Nasrolahpour, Prespacetime J. 2 (13) (2011) 2053-2059.

[4] H. Nasrolahpour, Prespacetime J. 3 (1) (2012) 99-108.

[5] H. Nasrolahpour, Prespacetime J. 3 (12) (2012) 1194-1196.

[6] H. Nasrolahpour, Prespacetime J. 3 (13) (2012) 1247-1250.

[7] H. Nasrolahpour, Prespacetime J. 4 (6) (2013) 604-608.

[8] V. E. Tarasov, G. M. Zaslavsky, Comm. Nonl. Sci. Num. Simul. 11 (2006) 885-898.

[9] N. Korabel et al. Comm. Nonl. Sci. Num. Simul. 12 (2007) 1405-1417.

[10] D. Baleanu et al., Nonlinear Dyn. 60 (2010) 81-86.

[11] D. Baleanu et al., Cent. Eur. J. Phys. 8(1) (2010) 120-125.

[12] Y. Luchko, J. Math. Phys. 54 (2013) 012111.

[13] S. I. Muslih et al., J. Phys. A: Math. Theor. 43 (2010) 055203.

[14] S. I. Muslih et al., Int. J. Theor. Phys. 49 (2010) 1746-1752.

[15] J. F. Gómez-Aguilar et al., Revista mexicana de fisica 58 (4) (2012) 348-352.

[16] J. F. Gómez-Aguilar et al., Journal of Electrical Bioimpedance 3 (1) (2012) 2-11.

[17] J. F. Gómez-Aguilar et al., Entropy 17 (9) (2015) 6289-6303.

[18] R. Hilfer, Applications of Fractional Calculus in Physics (World Scientific, 2000).

[19] J. Sabatier, et al. (Eds.), Advances in Fractional Calculus (Springer, 2007).

[20] R. Herrmann, Fractional Calculus (World Scientific Press, 2011).

[21] J. Klafter et al. (Eds.), Fractional Dynamics: Recent Advances (World Scientific, 2011).

[22] H. Nasrolahpour, Comm. Nonl. Sci. Num. Simul. 18(9) (2013) 2589-2593.

[23] M. D. Ortigueira, Fractional Calculus for Scientists and Engineers (Springer, 2011).

[24] V. V. Uchaikin, Fractional Derivatives for Physicists and Engineers (Springer, 2012).

[25] K. B. Oldham, J. Spanier, the Fractional Calculus (Academic Press, 1974).

[26] S. G. Samko et al., Fractional Integrals and Derivatives (Gordon and Breach, 1993).

[27] I. Podlubny, Fractional Differential Equations (Academic Press, 1999).

[28] M. Kaku, Quantum field theory, a modern introduction (Oxford University Press, 1993).
[29] M. Mathai, H. J. Haubold, Special Functions for Applied Scientists (Springer, 2008).

[30] M. Du et al., Sci. Rep. 3 (2013) 3431.

[31] A. Mvogo et al., Comm. Nonl. Sci. Num. Simul. 48 (2017) 258 269.

[32] A. Mvogo and T. C. Kofané, Chaos 26 (2016) 123120.

[33] J. A. Tenreiro Machado et al., Comm. Nonl. Sci. Num. Simul. 16 (2011) 2963-2969.

[34] H. Nasrolahpour, Fractional Field Theory Approach to Protein Folding Dynamics, bio Rxiv (2017) doi: 10.1101/153999.

[35] E. Ahmed et al, Journal of Fractional Calculus and Applications 3(2) (2012) 1- 6.

[36] F. A. Rihan et al., J. Tumor Res. 2(1) (2016) 109.

[37] X. Cao et al., J. Syst. Sci. Complex 29 (2016) 1565-1584.

[38] M. A. Moreles and R. Lainez, Commun Nonlinear Sci Numer Simulat 46 (2017) 81-88.

[39] W. Teka et al., PLOS Computational Biology 10(3) (2014) e1003526.

[40] M. Caputo and C. Cametti, Physica A 462 (2016) 705-713.

[41] Z. B. Vosika et al., PLOS ONE 8(4) (2013) e59483.

[42] C. M. A. Pinto and A. R. M. Carvalho, J. Comput. App. Math. 312 (2017) 240-256.

[43] H. Nasrolahpour, Fractional Dynamics in Bioscience and Biomedicine and the Physics of Cancer, bio Rxiv (2017) doi: 10.1101/214197.

[44] V. E. Tarasov, Fractional Dynamics (Springer, 2011). V. E. Tarasov, Int. J. Mod. Phys. A 27(9) (2013) 1330005.

[45] V. V. Uchaikin, Fractional Derivatives for Physicists and Engineers (Springer, 2012).

[46] K. B. Oldham, J. Spanier, the Fractional Calculus (Academic Press, 1974).

[47] S. G. Samko et al., Fractional Integrals and Derivatives (Gordon and Breach, 1993).

[48] I. Podlubny, Fractional Differential Equations (Academic Press, 1999).

[49] F. Mainardi, Chaos Solit. Fract. 7(9) (1996) 146.

[50] F. Mainardi et al., Fractional Calculus Appl. Anal. 4(2) (2001) 153.

[51] R. Meng et al., Applied Mathematical Modelling 40 (2016) 398-406.

[52] J. E. Palomares-ruiz et al., Revista Mexicana de Fisica 61 (2015) 261-267.

[53] N. Demirci and E. Tönük, Acta of Bioengineering and Biomechanics 16 (4) (2014) 13-21.

[54] B. Joźwiak et al., PLoS ONE (10) (2015) e 0143090.

[55] B. Carmichael, Phys. Biol. 12 (2015) 046001.

[56] C. A. M. La Porta and S. Zapperi, Physics of Cancer (Cambridge University Press, 2017). 